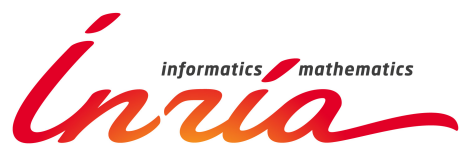

Deciding

Non-Compressible Blocks

in Sparse Direct Solvers

using Incomplete

Factorization

Esragul Korkmaz, Mathieu Faverge, Grégoire Pichon, Pierre Ramet

RESEARCH

REPORT

$\mathbf{N}^{\circ} 9396$

February 2021

Project-Team HIEPACS 



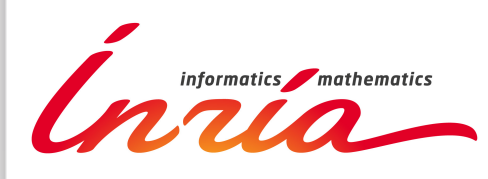

\title{
Deciding Non-Compressible Blocks in Sparse Direct Solvers using Incomplete Factorization
}

\author{
Esragul Korkmaz朋, Mathieu Faverge ${ }^{\dagger \star * \S}$, Grégoire Pichon] \\ Pierre Ramet $\$ \ddagger *$
}

Project-Team HIEPACS

Research Report n 9396 - February 2021 - 16 pages

\begin{abstract}
Low-rank compression techniques are very promising for reducing memory footprint and execution time on a large spectrum of linear solvers. Sparse direct supernodal approaches are one these techniques. However, despite providing a very good scalability and reducing the memory footprint, they suffer from an important flops overhead in their unstructured low-rank updates. As a consequence, the execution time is not improved as expected. In this paper, we study a solution to improve low-rank compression techniques in sparse supernodal solvers. The proposed method tackles the overprice of the low-rank updates by identifying the blocks that have poor compression rates. We show that block incomplete LU factorization, thanks to the block fill-in levels, allows to identify most of these non-compressible blocks at low cost. This identification enables to postpone the low-rank compression step to trade small extra memory consumption for a better time to solution. The solution is validated within the PASTIX library with a large set of application matrices. It demonstrates sequential and multi-threaded speedup up to $8.5 x$, for small memory overhead of less than $1.49 x$ with respect to the original version.
\end{abstract}

Key-words: Sparse direct solvers, low-rank compression, ILU factorization

* Inria Bordeaux - Sud-Ouest, Talence, France

$\dagger$ Bordeaux INP, Talence, France

$\ddagger$ CNRS (Labri UMR 5800), Talence, France

$\S$ University of Bordeaux, Talence, France

I Univ Lyon, EnsL, UCBL, CNRS, Inria, LIP 


\section{Détecter les blocs non compressibles dans les solveurs directs creux par une factorisation incomplète}

Résumé : Les techniques de compression de rang faible sont très prometteuses au niveau de l'empreinte mémoire et du temps d'exécution sur un large spectre de solveurs linéaires. Les approches supernodales directes creuses font partie de ce spectre. Cependant, malgré leur très bonne scalabilité et leur empreinte mémoire plus faible, elles souffrent d'un surcoût calculatoire important dû aux mises à jour non-structurées de rang faible impliquées. En conséquence, le temps d'exécution n'est pas amélioré comme prévu. Dans cet article, nous étudions une solution pour améliorer les techniques de compression de rang faible dans les solveurs supernodaux creuses. La méthode proposée s'intérresse à ces mises à jour de rang faible plus coûteuses en identifiant les blocs avec de faibles taux de compression. Nous montrons que la factorisation LU incomplète par blocs, grâce aux niveaux de remplissage des blocs, permet d'identifier, à faible coût, la plupart de ces blocs non compressibles. Cette identification permet de reporter l'étape de compression de rang faible pour permettre un meilleur temps de résolution en échange d'une légère sur-consommation mémoire. La solution est validée dans la bibliothèque PaStiX avec un large ensemble de matrices issues d'applications. Il démontre une accélération séquentielle et multithread jusqu'à $8.5 \mathrm{x}$, pour une augmentation de la consommation mémoire inférieure à $1.49 \mathrm{x}$ par rapport à la version originale.

Mots-clés : Solveur direct creux, compression de rang faible, factorisation ILU 


\section{Introduction}

In many engineering and scientific applications, solving a large sparse linear system of the form $A x=b$ is a mandatory but very time consuming step. Among the many various approaches to solve these systems, sparse direct solvers [9] are a robust and widely used solution. However, they are known to be both time and memory consuming. Recent studies tackle these issues by experimenting different data-sparse compression schemes trading a controlled precision loss for better memory and computation complexities. These techniques include but are not limited to (Multilevel-)Block Low-Rank format (BLR) 1, 3, 21, $\mathcal{H}$ Matrices 11, 17, $\mathcal{H}^{2}$ 13, Hierarchical Off-Diagonal Low-Rank (HODLR) [4, 6] or Hierarchically Semi-Separable (HSS) [10, 25]. These techniques can be classified into two categories. The first one considers the full problem and extracts the sparsity of the matrix from the low-rank representation. The second solution exploits the existing sparsity of the matrix structure and compresses the blocks that compose it independently. This paper focuses on the latter. More specifically, it targets block low-rank methods (BLR).

In this context, as in other linear algebra solvers, one of the most important operation is the block update. When using regular block sizes, as in dense or sparse multifrontal solvers, the cost of the low-rank update is usually small with respect to the full-rank version. On the other hand, when various block sizes are involved, as in sparse supernodal solvers, the cost of the low-rank update depends on the largest block involved. Therefore, the update operation may become more expensive than the full-rank one. However, supernodal approaches provide more parallelism and have less memory overhead than multifrontal methods. As a consequence, in this paper, we propose to improve supernodal methods by trading a small memory overhead for lower flop count and better time to solution. For that purpose, we implement our solution in the sparse direct solver PASTiX [20, 21], which supports the BLR compression scheme.

The PAStiX solver offers two opposite strategies: favor memory peak reduction over time to solution (Minimal Memory), or prefer time to solution by delaying the data compression (Just-In-Time). The former suffers from the costly update operations, while the latter avoids it without reducing the memory consumption compared to the full-rank solver. Identifying the potential compressibility of each block is a key problem to benefit from both strategies. In this work, we propose a new technique based on incomplete factorization to define levels of admissibility (compressibility) for the blocks. The algorithm is applied during the preprocessing stage and provides a trade-off between memory and flops. We show that this solution, while targeting the Minimal Memory strategy, may also improve the Just-In-Time solution.

Section 2 sets the basis of this work by giving background information on the BLR implementation within the PASTIX solver and its limitations, as well as introducing the block incomplete $L U$ factorization. Section 3 presents the related work. Section 4 details the new heuristic, which defines the non-compressible blocks to exploit the existing compression strategies in PASTIX. Section 5 anal- 
yses experiments on a large set of matrices. Finally, Section 6 concludes on the results of this work and its perspectives.

\section{Background}

This section provides background details on the PAStiX solver and its BLR implementation and recalls the general idea behind incomplete LU factorization.

\subsection{Sparse supernodal direct solver using BLR compres- sion}

Sparse direct solvers generally follow four steps: 1) order the unknowns to reduce the fill-in that occurs during factorization; 2) perform a block-wise symbolic factorization to compute the structure of the final factorized matrix; 3) compute the actual numerical factorization based on the structure resulting from step 2; and 4) solve the triangular systems. In the remainder of the paper, we will focus only on the numerical factorization (step 3) and the matrix values initialization. We consider that steps 1 and 2 already exhibit a structure with large enough blocks to take advantage of BLAS Level 3 [8 operations whenever possible. Step 4 is not influenced by the work presented in this paper, and is thus not further discussed.

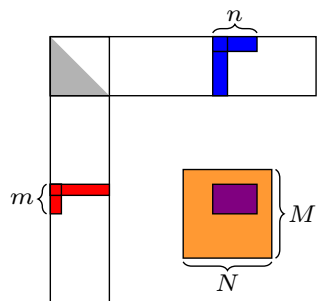

(a) Non-fully structured update (LR2FR)

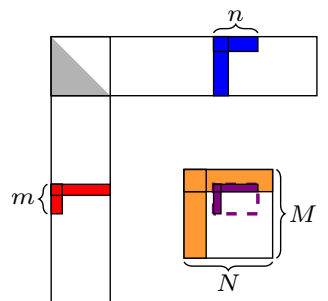

(b) Fully structured update (LR2LR)

Figure 1: Representation of the updates, $C-=A B$, for the Just-In-Time strategy on the left, and Minimal Memory on the right. $A$ appears in red, $B$ in blue, $C$ in orange, and the impact of the contribution $A B$ in purple.

BLR compression scheme has been introduced in the sparse supernodal solver PaStiX in [20, 21. As already mentioned, the solver implements two strategies to target either lower memory cost (Minimal Memory), or lower time to solution (Just-In-Time). Both strategies differ depending on the update kernels, $C=C-A B$, that are involved in the numerical factorization. Figure 1 describes both options. On the left, the non-structured updates (LR2FR) lower the cost of the matrix product $A B$ thanks to the low-rank representation. However, the updated $C$ matrix is stored in full-rank to be able to perform a simple addition of the contribution at a cost of order $m n$, with $m$ and $n$ the 
dimensions of this contribution. On the right, although the low-rank structured update (LR2LR) is more complex, the $C$ matrix is stored in low-rank to save memory. Here, while the contribution $(A B)$ is also computed at a lower cost, the addition step into $C$ requires a more complex low-rank to low-rank update with padding (zeroes are added to match the dimension of $C$ ). This update has a complexity of order $M N$, with $M$ and $N$ the dimensions of the updated matrix $C$. A more detailed complexity analysis is provided in [21].

The Just-In-Time strategy aims only at reducing the time to solution by exploiting the LR2FR update kernel. Indeed, LR2FR relies on high performance matrix-matrix multiplication kernels with smaller sizes than the ones of the full-rank implementation. On the other hand, the Minimal Memory strategy compresses the matrix at initialization exploiting the graph of the matrix to speedup the compression. Blocks of the factorized matrix which do not hold initial information are null and thus compressed at no cost. Other initial blocks compression can also be accelerated by automatically removing null columns and rows from the graph knowledge. This operation greatly improves the memory peak of the solver as the factorized matrix structure is never fully allocated. However, it forces the numerical factorization to rely on the LR2LR kernel. This kernel in the context of the supernodal method generates a flop overhead due to the padding operation. As a consequence, the factorization time may be highly impacted, unless the matrix is highly compressible. Thus, deciding which blocks to compress and when to do it is an important problem for supernodal solvers to reach good level of performance while benefiting from the lower memory consumption offered by low-rank techniques. Note that in sparse direct solvers, only blocks with sufficiently large sizes are considered to be admissible for lowrank compression. All small blocks are automatically defined as non-admissible due to their lower impact.

To decide which blocks to compress, 12 defined the admissibility condition. More specifically, the study proposes a strong and a weak admissibility conditions. The strong admissibility condition relies on the problem geometry and the definition of the diameter of a set of unknowns $(\operatorname{diam}(\sigma))$, as well as the distance between two sets $(\operatorname{dist}(\sigma, \tau))$. The interaction between two sets of unknowns is then considered admissible if the distance between the sets is sufficiently larger than both of the diameters of the sets $(\max (\operatorname{diam}(\sigma), \operatorname{diam}(\tau)) \leq \eta \operatorname{dist}(\sigma, \tau))$. The least restrictive strong admissibility criterion considers that blocks are admissible only if their distance is larger than 0. Thus, all blocks except close neighbors are compressible. On the other hand, the weak criterion simply considers that all non-diagonal blocks are compressible, which is equivalent to the Minimal Memory strategy. This paper proposes to generalize the distance criterion used in the strong admissibility condition by computing algebraic distances of the blocks without any geometry knowledge requirement. The distance computation is performed with block-wise ILU factorization. 


\subsection{Incomplete LU factorization}

The incomplete LU (ILU) factorization is an approximated version of the LU factorization, where part of the information is dropped 23. It has the form $A \approx L U=L U+R$. Here, the matrix $\mathrm{R}$ carries the negative values of the dropped elements. This method is usually used as a preconditioner for iterative methods [14.
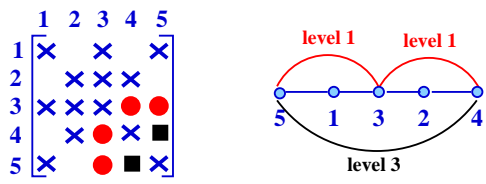

Figure 2: An adjacency matrix (on the left) and its associated graph (on the right). Fill-in entries that may occur during the numerical factorization are represented in red (level 1) and black (level 3).

Numerical or graph constraints are applied to obtain the ILU factorization. For example, the dropping can be based on a threshold [16, or on the fill-in levels [5]. We consider only the latter in this paper. The ILU method with the fill-in levels definition as we use was first suggested in 1981 24] and then improved by a graph-based definition in [15, 22. Figure 2 illustrates the idea of the fill-in levels that is strongly related to the ordering of the unknowns. On the left, the matrix non-zeroes pattern is represented, where the blue crosses are the original entries. On the right, the graph associated to this matrix is shown. During the numerical factorization, some entries may become non-zeroes (fillin). On Figure 2, these entries are represented in red and black, both on the matrix and as new edges on the graph. We can define the fill-in level as the length of the path connecting the two unknowns in the original graph. The path connecting 3 and 5 (and 3 and 4) in red goes only through 1 (resp. 2). Thus, the level of the fill-in between these two unknowns is 1 . We can also see that 4 and 5 are connected at a level 3 (the path goes through 1, 3 and 2). As the fill-in level gets higher, the value of the new entry becomes smaller as it represents far interactions in the graph. Therefore, the ILU factorization can be implemented by omitting the values which have higher fill-in levels than a predefined maximum level. Similarly, this procedure can be applied in a blockwise fashion. Thus, the block fill-in level can be considered as an admissibility criterion for low-rank compression.

\section{Related work}

Many recent studies have tackled the problem of reducing the memory consumption of linear solvers with low-rank compression.

In [12, the adaptation of hierarchical matrix techniques issued from the dense community to sparse matrices is studied. Here, by ignoring some structural zeroes, the opportunity for further memory savings is missed. Although 
low-rank updates are performed similarly to the Minimal Memory strategy, padding is not used as the zeroes are explicitly stored. This results in more efficient updates at the cost of a larger memory consumption.

Low-rank updates in the context of sparse supernodal solvers have already been studied in 6. In this work, the authors considered fixed ranks for the blocks which must be known in advance. They demonstrated interesting memory savings, but with a slower factorization than the full-rank version.

The sparse multifrontal BLR solver MumPs [2, 19] implements two similar solutions: CUFS (Compress, Update, Factor, Solve) which is similar to our Minimal Memory scenario, and FCSU which is closer to the Just-In-Time scenario. However, as it is a multifrontal solver, these strategies are applied with tiled algorithms on the dense matrices of the fronts that appear during the factorization. Here, the memory saving is limited as fronts are allocated in full-rank before being compressed.

In [18, the preselection problem is approached from a different angle. The authors exploit performance models of the update kernel to decide whether or not to delay the compression of some of the blocks. This decision is taken at runtime during the numerical factorization and requires to generate correct models of the problem.

\section{Deciding the Non-Compressible Blocks}

As mentioned in Section 2, the Minimal Memory strategy suffers from the complexity overhead of the LR2LR update kernel. We propose to exploit ILU fill-in levels to identify, at low cost, the blocks with large ranks. These blocks will increase the cost of the update step while providing only a small memory reduction. Once identified, it is possible to postpone the compression of these blocks as late as possible to replace the LR2LR kernels by LR2FR. This comes to the cost of a controlled memory overhead if the identification is correct. Section 2.2 recalls that as the ILU fill-in levels get larger, the magnitude of the entries gets smaller. Thus, blocks with large level values should have small ranks and should be kept compressed to save memory, while blocks with small level values should have high ranks.

Algorithm 1 presents the main steps to compute the fill-in levels of the blocks. This algorithm performs the same loops as the numerical factorization focusing only on the fill-in level information and the symbolic structure of the factorized matrix $L$. Initially, all blocks are considered with level 0 , if they are part of the original matrix $A$, or $\infty$ if they are created by fill-in. Then, the main loop updates the levels according to the formula given in 23, which is adapted to the block-wise algorithm (Line 7). Note that, as PASTiX uses the symmetric pattern structure of $A+A^{T}$ even for $L U$ factorization, Choleskybased algorithms are presented. For strongly non-symmetric matrices, the fill-in levels of the blocks in $L$ and $U$ are computed separately for better identification. This very cheap algorithm can be interleaved with the matrix initialization to completely hide its cost.

$\mathrm{RR} \mathrm{n}^{\circ} 9396$ 

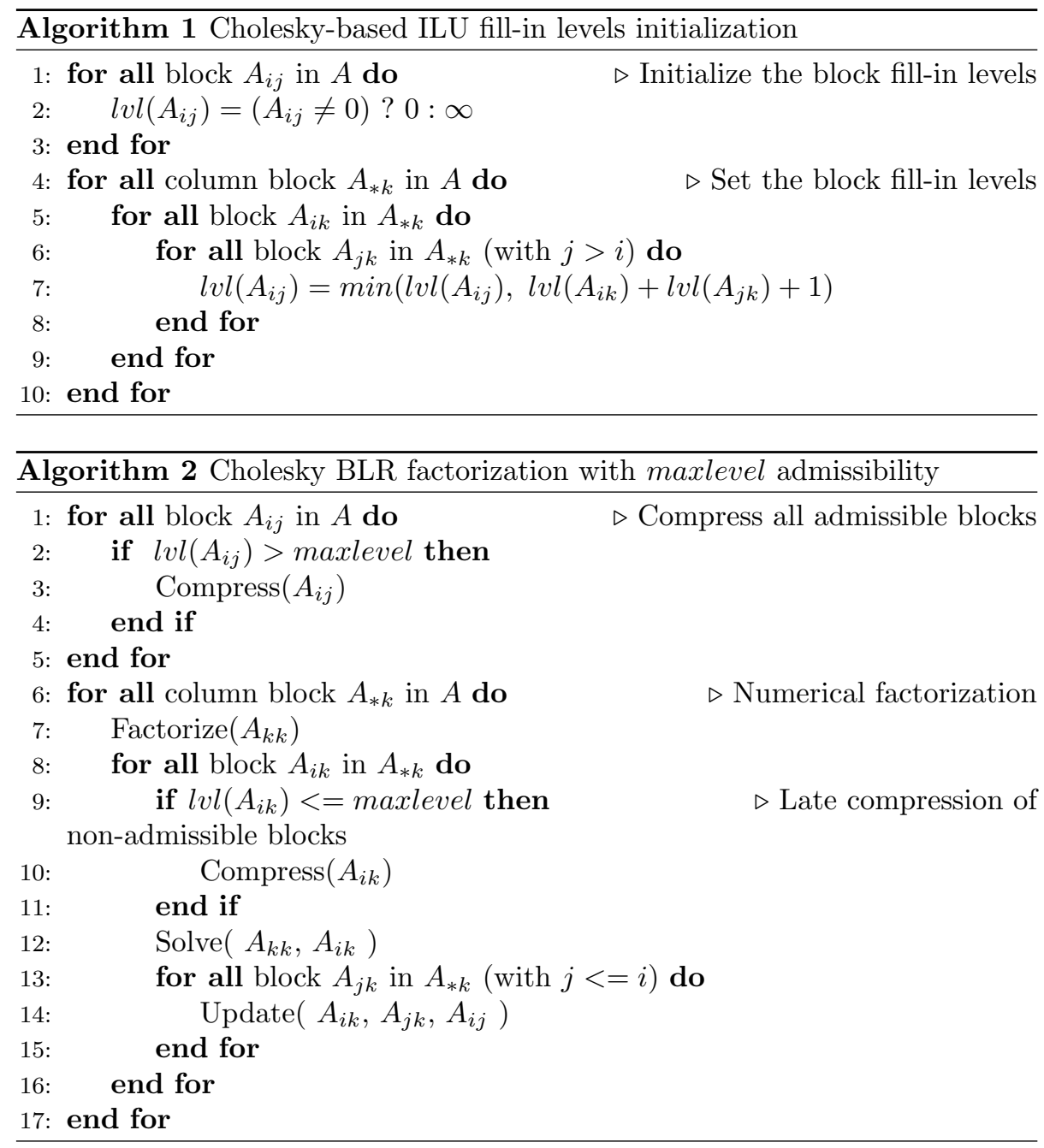

Now that the fill-in levels are computed, the numerical factorization can be adapted to exploit this information. Algorithm 2 presents the proposed algorithm with a generic parameter maxlevel, which allows to set the new admissibility criterion. First, lines $1-5$ compress the admissible blocks which have a fill-in level larger than maxlevel. These blocks have the smallest ranks. Thus, they will be involved in LR2LR updates and are the most important ones to compress to reduce the memory footprint. On the other hand, the nonadmissible blocks will be involved in LR2FR updates to reduce the flop count overhead while inducing a small memory overhead. These blocks are still compressed, just after the factorization of the diagonal block, to reduce the cost of the following operations: solve and updates. In the remainder of the paper, we will refer to this BLR sparse factorization as $I L U(k)$, with $k$ the maximum 
level of the admissibility criterion. Note that choosing the right $k$ value for a given problem is important. As a matter of fact, the larger the number of non-admissible blocks, the higher the memory overhead.

It is important to observe that ILU(-1) is the Minimal Memory scenario as all the admissible blocks are compressed during the initialization. On the opposite, ILU $(\infty)$ corresponds to the Just-In-Time scenario as all blocks are compressed only after all the updates were accumulated.

\section{Experiments}

All the experiments are performed through the BLR supernodal direct solver PaStiX [21, using the miriel nodes of the Plafrim 1 supercomputer. Each miriel node is equipped with two INTEL Xeon E5-2680v3 12-cores running at $2.50 \mathrm{GHz}$ and $128 \mathrm{~GB}$ of memory. For the multithreaded experiments, we use 24 threads, one per core, on these nodes. The INTEL MKL 2018 is used for the BLAS kernels. We set the limits to allow the compression to 128 for the block width and 20 for the height. The minimum block width and height criteria to allow compression are 128 and 20, respectively. In the experiments, we use only $L D L^{T}$ and $L U$ factorizations according to the input matrix features. For the SPD matrices, we avoid $L L^{T}$ factorization as the positive definite feature can be affected by the compression. In the following sections all the experiments are run for a set of 31 real case matrices taken in the SuiteSparse Matrix Collection [7]. Data reported in the graphs are related to the numerical factorization only. The solve step is never considered as it is not impacted by this new algorithm. The times shown are the average of 3 runs on each matrix.

\subsection{Compressibility Statistics}

We first want to validate the hypothesis that using the fill-in levels is a good heuristic to classify the blocks based on their compressibility ratios. Figure 3 reports the accumulated memory consumption for all the 31 studied matrices in our experiments with three different tolerance criteria and by fill-in levels. The purple bars show the memory consumption of the structurally non-admissible blocks (too small to be compressed) and is stable for all precisions. The red part corresponds to the admissible blocks. The dark red is the memory footprint when they are compressed. It naturally increases with a more accurate tolerance. The light red shows the amount of memory that can be saved by compressing the blocks. One can observe that the lower the tolerance, the higher the gain.

These results show that the compression ratio of the admissible blocks increases with the levels. It confirms the original hypothesis, that fill-in levels can help to better tune the admissibility criterion in order to save flops for a small memory overhead. Furthermore, this parameter needs tuning to adapt to the tolerance. One can see that for a tolerance of $1 e^{-12}$, only levels greater than 3 offer more than $50 \%$ memory savings, while all levels at $1 e^{-4}$ almost reach $50 \%$

\footnotetext{
1 https://www.plafrim.fr
} 


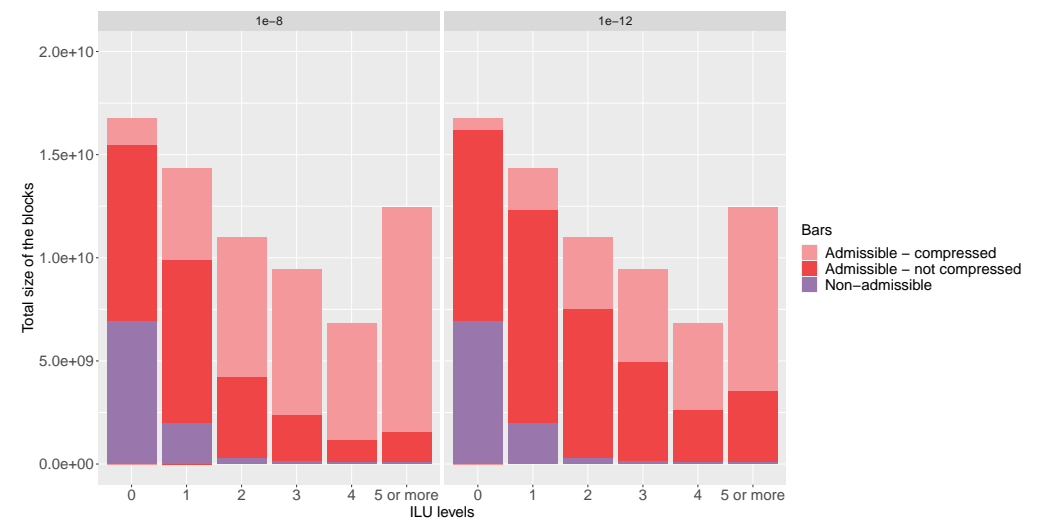

Figure 3: Potential memory saving based on the tolerance criterion and fill-in levels. The bars report the cumulative memory of the 31 matrices. Purple represents the memory consumed by blocks below the size criteria, and red the memory of the admissible blocks for compression. Light red is the portion that can be saved when compressed.

savings. As a consequence, the fill-in level used to define the admissibility criterion will need to be adapted to both the tolerance and the maximum memory overhead defined by the user.

\subsection{Comparison of the Costs}

This section discusses the sequential experiments. Figure 4 shows the memory peak, factorization flops and factorization time profiles obtained for different precisions. We study the impact of the first fill-in levels (0 to 4$)$ with respect to Minimal Memory $(-1)$ and Just-In-Time $(\infty)$. Each curve represents the number of matrices within a percentage overhead of the best solution for each metric and matrix.

First, as expected, the lower the fill-in level chosen for admissibility, the lower the memory peak of the solver. One can observe that the impact of the fill-in level increases as the accuracy decreases. This confirms the trend already observed on Figure $3 . I L U(\infty)$ consumes up to 6.6 times more memory at $1 e^{-4}$ while it drops to 3.4 times at $1 e^{-12}$. Additionally, at this high tolerance, high levels of fill-in are able to reach the best memory peak. That means that potential flops reduction is possible without negatively impacting the memory.

Second, when observing the flop count evolution, the results are naturally reversed. The higher levels of fill-in are better to generate less flops. One can observe that for low accuracy $\left(1 e^{-4}\right)$, a level of 0 is enough to reach the same flop count as the Just-In-Time scenario $(I L U(\infty))$. When increasing the accuracy, more levels need to be considered admissible to lower the flop count to its minimal value. Except some corner cases, levels 1 or 2 are enough for 
$1 e^{-8}$, and respectively 3 or 4 for $1 e^{-12}$.

Finally, the time profiles follow the same trend as the flops profiles, with larger differences between the $I L U(k)$ methods. This can be explained by the disparity of the LR2LR and LR2FR efficiency, as well as their variation in number that may increase the phenomena already observed on flops. Thus, one can observe that $I L U(0)$ is the best average solution at $1 e^{-4}$. It even outperforms the Just-In-Time strategy by a factor up 1.4x. To explain this performance, we recall that in the Just-In-Time strategy, many null-rank blocks are allocated and later compressed, while in the new $I L U(k)$ heuristic they may never be allocated. Indeed, they are originally null blocks and at low precision they may receive only null contributions. Thanks to these savings, $I L U(0)$ at this tolerance almost doubles the memory footprint in the worst case with respect to the Minimal Memory strategy, but it remains 0.23 times the memory consumption of the $I L U(\infty)$ and the full-rank versions.

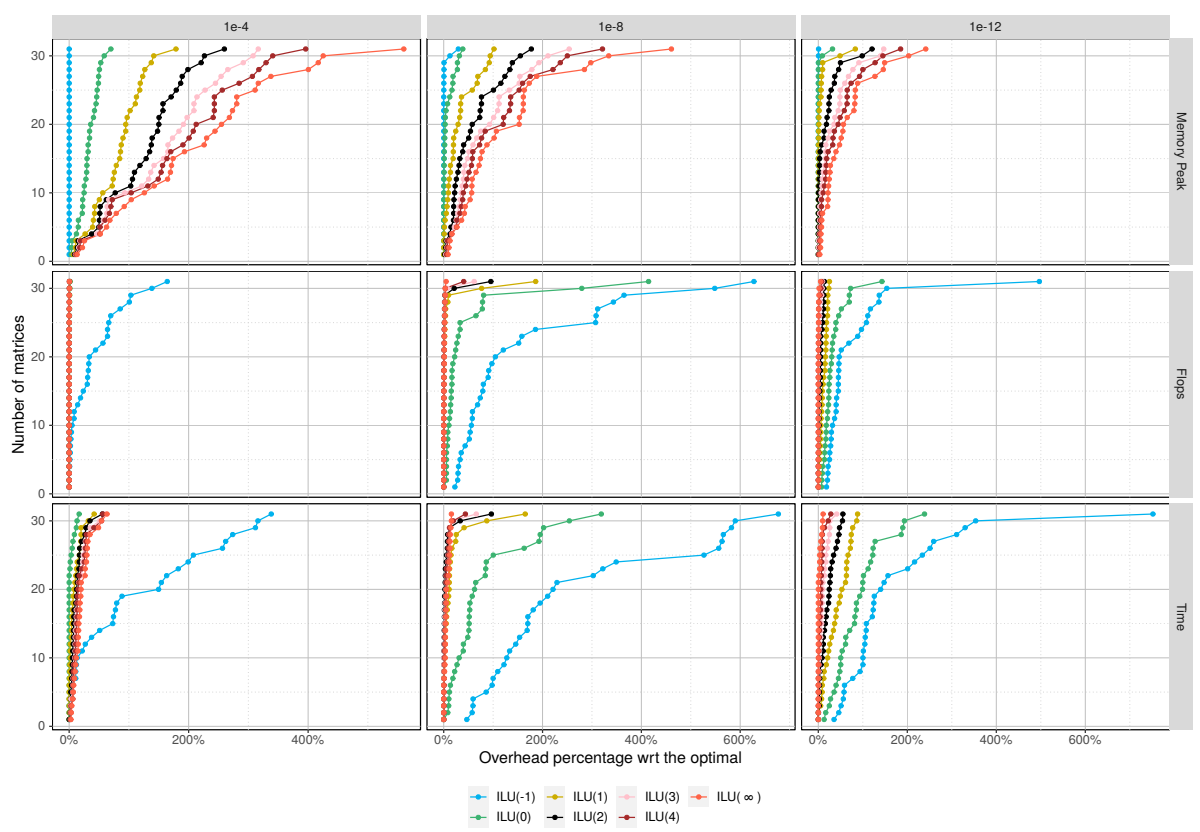

Figure 4: Memory peak, factorization flops and factorization time profiles of different precisions with sequential runs. Each color stands for a different $I L U(k)$ level. The $\mathrm{x}$-axis shows percentages with respect to the best method for each metric and matrix, while y-axis represents the matrix count in accumulated way.

The observations in higher precisions are similar to the low precision, but with higher fill-in levels. At $1 e^{-8}$, only the levels above 1 compete with the JustIn-Time strategy in terms of time, as well as providing a controlled memory overhead with respect to the best solution.

At $1 e^{-12}$, the levels higher than 3 are required to get the best time to solu- 
tion, which reduces the gain one can obtain on the memory footprint. However, solutions with level 1 is a good compromise at this precision as it gets similar memory footprints as $I L U(-1)$ (up to $1.49 x$ ), while being up to 8.5 times faster.
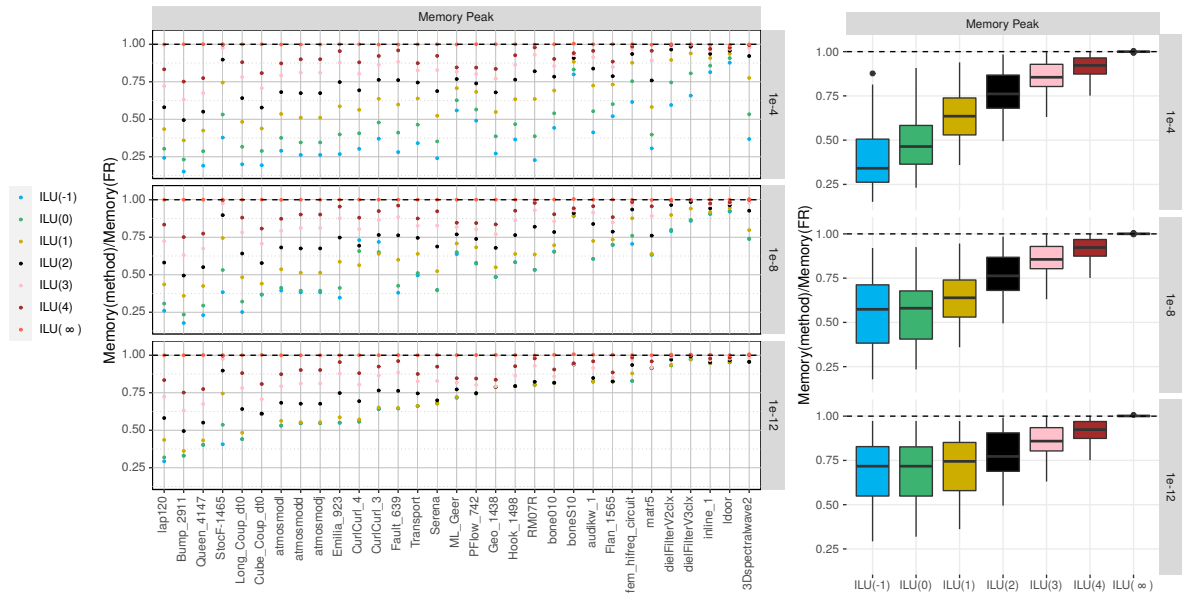

Figure 5: Memory peak ratio of the $I L U(k)$ heuristic with respect to full-rank on the 31 test matrices. On the left, the detailed information is presented. On the right, the information is summarized with boxplots showing minimum, maximum, median, first quartile and third quartile of each level.

Figure 5 presents the detailed ratios of the memory peak of the different levels of admissibility with respect to the full-rank solution. One can observe that the Just-In-Time $(I L U(\infty))$ strategy has the same memory peak as the full-rank version, and that the lower the level, the lower the memory peak. As we can observe all the matrices separately on the left, we can characterize them to decide which $I L U(k)$ level is more convenient for each matrix to minimize the memory footprint. For example, one can see that, at $1 e^{-12}$, the highest level giving the minimal memory footprint is 1 for the first half, and 2 for the second half. These trends are similar on the flops and time figure showing that they are strongly connected. One can also observe, at $1 e^{-4}$ that as mentioned above, increasing the level of admissibility seems to greatly increase the memory footprint with respect to the best one. However, it remains moderate when comparing with the full-rank implementation and it can be afforded to greatly reduce the time to solution.

The summary on the right of Figure 5 confirms the fact that the memory consumption increases with the higher fill-in levels and with the higher precision. The results at $1 e^{-12}$ for the new heuristic at levels 1 and 2 show interesting results. As a matter of fact, they provide $25 \%$ memory improvement compared to the full rank version with equivalent time to solution. Note that, at this precision, even $I L U(\infty)$ does not manage to accelerate the full-rank version on the five most-right cases which do not compress at all. On the others, levels 1 
and 2 are the best compromises since they are faster than the full-rank version while providing $25 \%$ memory saving in average.

To conclude, it is difficult to give one level as the optimal solution, but depending on the problem and the precision required, the level can be tuned to provide a solution that outperforms the Minimal Memory strategy in terms of time to solution for a small controlled memory overhead. Moreover, it can even have a speedup compared to the Just-In-Time strategy with less memory footprint.

\subsection{Gain for the Multithreaded Version}

This section presents the same experiments as in the previous section in a multithreaded environment with 24 threads. Figure 6 presents the time profiles of the multi-threaded numerical factorization. Memory peak and flops are not reported as they are identical to the sequential ones.

We can observe that the effects already seen in sequential are also observed in a multi-threaded environment with a larger impact of the changes in the kernels (LR2LR or LR2FR). $I L U(k)$ performs better with a level of 0,2 and 4 , for tolerances of respectively $1 e^{-4}, 1 e^{-8}$, and $1 e^{-12}$. Here, the Just-In-Time strategy is losing even more with respect to the $I L U(k)$ strategy as it can be especially seen at $1 e^{-4}$. In this case, we can also consider that the large memory reduction impacts the memory accesses of the threads. It helps $I L U(0)$, which initially stores only the blocks of the original matrix $A$, to outperform the other versions. At $1 e^{-12}$, one can observe that choosing $I L U(2)$ is a good competitor to $\operatorname{ILU}(\infty)$, while saving $25 \%$ of memory in average.

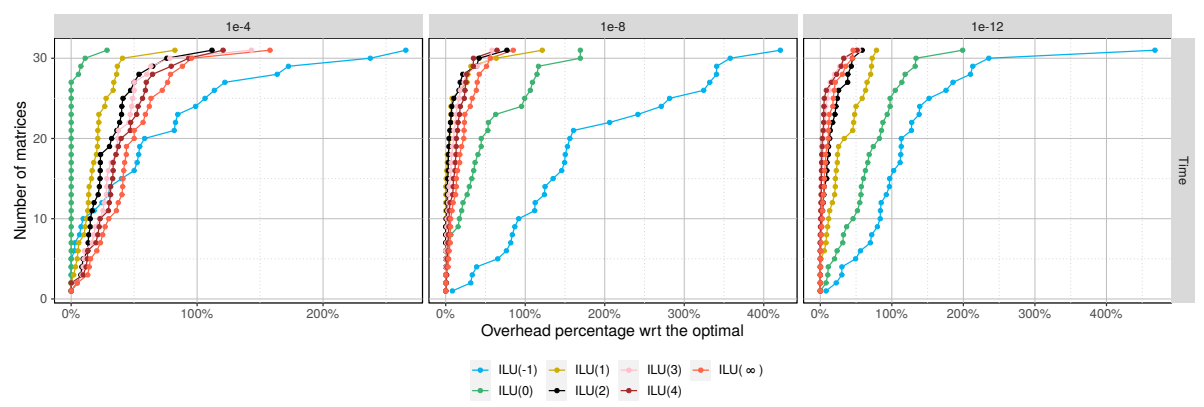

Figure 6: Time profiles of the multithreaded runs for different precisions. Each color shows a different ILU(k) level result.

\section{Conclusion}

The behavior of sparse supernodal direct solvers using low-rank compression highly depends on when the compression is performed. On one hand, all admissible blocks can be compressed before the factorization (as it happens with the

$\operatorname{RR} n^{\circ} 9396$ 
Minimal Memory strategy), allowing high memory savings at the cost of expensive low-rank updates. On the other hand, admissible blocks can be compressed after they have received all their updates (as for the Just-In-Time strategy), reducing significantly execution time with a controlled memory overhead.

In this paper, we proposed a new heuristic to estimate the compressibility of each block and constructed an algorithm that is a compromise between the two strategies mentioned previously. The new heuristic, named $I L U(k)$, relies on the ILU fill-in levels to define an algebraic distance to compute low-rank admissibility of the blocks. The purpose of defining the admissibility was to propose an intermediate solution that accelerates the Minimal Memory solution while it slightly increases the memory consumption.

The experiments that we conducted on a large set of 31 real matrices demonstrated that our heuristic, $I L U(k)$, manages to identify the low-rank blocks efficiently. The solution proposed runs up to 8.52 times faster than Minimal Memory with only a 1.49 times increase of memory usage for high precisions in both sequential and multi-threaded environments. Moreover, due to the elimination of the null blocks before the numerical factorization, our new heuristic is able to run 1.4 times faster than the Just-In-Time strategy in sequential, with a much lower memory consumption (0.23 times less). In the multi-threaded environment, it even goes to 2.01 times faster for most of the cases. However, the best $k$ value to use is not defined, even if clear trend appears.

For future work, we plan to introduce tuning techniques to automatically infer the best value of $k$ depending on the properties of the machine used and the tolerance given. An orthogonal work of this paper consists in exhibiting more regular sparse structures, gathering unknowns that receive similar contributions (and thus have the same fill-in level). It could be done for instance by aligning separators, as presented in [20].

\section{Acknowledgments}

This work is supported by the Agence Nationale de la Recherche, under grant ANR-18-CE46-0006 (SaSHiMi). Experiments presented in this paper were carried out using the PlaFRIM experimental testbed, supported by Inria, CNRS (LABRI and IMB), Université de Bordeaux, Bordeaux INP and Conseil Régional d'Aquitaine (https://www.plafrim.fr/).

\section{References}

[1] Amestoy, P.R., Ashcraft, C., Boiteau, O., Buttari, A., L'Excellent, J.Y., Weisbecker, C.: Improving Multifrontal Methods by Means of Block Low-Rank Representations. SIAM Journal on Scientific Computing 37(3), A1451-A1474 (2015)

[2] Amestoy, P.R., Buttari, A., L'Excellent, J.Y., Mary, T.: On the complexity of the block low-rank multifrontal factorization. SIAM Journal on Scientific 
Computing 39(4), 1710-1740 (2017). https://doi.org/16M1077192, https : //oatao.univ-toulouse.fr/19066/

[3] Amestoy, P.R., Buttari, A., L'Excellent, J.Y., Mary, T.: Bridging the gap between flat and hierarchical low-rank matrix formats: the multilevel BLR format. SIAM Journal on Scientific Computing 41(3), A1414-A1442 (May 2019)

[4] Aminfar, A., Darve, E.: A fast, memory efficient and robust sparse preconditioner based on a multifrontal approach with applications to finiteelement matrices. International Journal for Numerical Methods in Engineering 107(6), 520-540 (2016)

[5] Campbell, Y., Davis, T.A.: Incomplete lu factorization: a multifrontal approach. https://ufdcimages.uflib.ufl.edu/UF/00/09/53/48/00001/1995185.pdf (October 1995)

[6] Chadwick, J.N., Bindel, D.S.: An Efficient Solver for Sparse Linear Systems Based on Rank-Structured Cholesky Factorization. CoRR abs/1507.05593 (2015)

[7] Davis, T.A., Hu, Y.: The University of Florida sparse matrix collection. ACM Trans. Math. Softw. 38(1), 1:1-1:25 (Dec 2011). https://doi.org/10.1145/2049662.2049663

[8] Dongarra, J., Du Croz, J., Hammarling, S., Duff, I.S.: A set of level 3 basic linear algebra subprograms. ACM Trans. Math. Softw. 16(1), 1-17 (1990)

[9] Duff, I.S., Erisman, A.M., Reid, J.K.: Direct methods for sparse matrices. Clarendon Press (1986)

[10] Ghysels, P., Li, X.S., Rouet, F.H., Williams, S., Napov, A.: An Efficient Multicore Implementation of a Novel HSS-Structured Multifrontal Solver Using Randomized Sampling. SIAM Journal on Scientific Computing 38(5), S358-S384 (2016)

[11] Hackbusch, W.: A Sparse Matrix Arithmetic Based on $\mathcal{H}$-Matrices. Part I: Introduction to $\mathcal{H}$-Matrices. Computing 62(2), 89-108 (1999)

[12] Hackbusch, W.: Hierarchical Matrices: Algorithms and Analysis, vol. 49 (12 2015). https://doi.org/10.1007/978-3-662-47324-5

[13] Hackbusch, W., Börm, S.: Data-sparse Approximation by Adaptive $\mathcal{H}^{2}$ Matrices. Computing 69(1), 1-35 (2002)

[14] Hénon, P., Ramet, P., Roman, J.: On finding approximate supernodes for an efficient block-ilu(k) factorization. Parallel Computing 34(6), 345-362 (2008). $\quad$ https://doi.org/https://doi.org/10.1016/j.parco.2007.12.003, https://www.sciencedirect.com/science/article/pii/ S0167819107001330, parallel Matrix Algorithms and Applications

$\mathrm{RR} n^{\circ} 9396$ 
[15] Hysom, D., Pothen, A.: A scalable parallel algorithm for incomplete factor preconditioning. SIAM J. Sci. Comput. 22, 2194-2215 (2001)

[16] Karypis, G., Kumar, V.: Parallel threshold-based ilu factorization. proceedings of the IEEE/ACM SC97 Conference (1997)

[17] Lizé, B.: Résolution directe rapide pour les éléments finis de frontière en électromagnétisme et acoustique : H-matrices. parallélisme et applications industrielles. Ph.D. thesis, École Doctorale Galilée, Paris, France (Jun 2014)

[18] Marchal, L., Marette, T., Pichon, G., Vivien, F.: Trading Performance for Memory in Sparse Direct Solvers using Low-rank Compression. Research Report RR-9368, INRIA (Oct 2020), https://hal.inria.fr/ hal-02976233

[19] Mary, T.: Block Low-Rank multifrontal solvers: complexity, performance, and scalability. Ph.D. thesis, Toulouse University, Toulouse, France (Nov 2017)

[20] Pichon, G.: On the use of low-rank arithmetic to reduce the complexity of parallel sparse linearsolvers based on direct factorization technique. Ph.D. thesis, Universite de Bordeaux,, Bordeaux, France (2018)

[21] Pichon, G., Darve, E., Faverge, M., Ramet, P., Roman, J.: Sparse supernodal solver using block low-rank compression: Design, performance and analysis. International Journal of Computational Science and Engineering 27, $255-270$ (Jul 2018)

[22] Rose, D.J., Tarjan, R.E.: Algorithmic aspects of vertex elimination on directed graphs. SIAM J. Appl. Math. 34(1), 176-197 (Jan 1978). https://doi.org/10.1137/0134014

[23] Saad, Y.: Iterative Methods for Sparse Linear Systems. Society for Industrial and Applied Mathematics, USA, 2nd edn. (2003)

[24] Watts, J.W.: A conjugate gradient truncated direct method for the iterative solution of the reservoir simulation pressure equation. Society of Petroleum Engineers Journal 21, 345-353 (1981)

[25] Xia, J.L.: Randomized sparse direct solvers. SIAM Journal on Matrix Analysis and Applications 34(1), 197-227 (2013) 


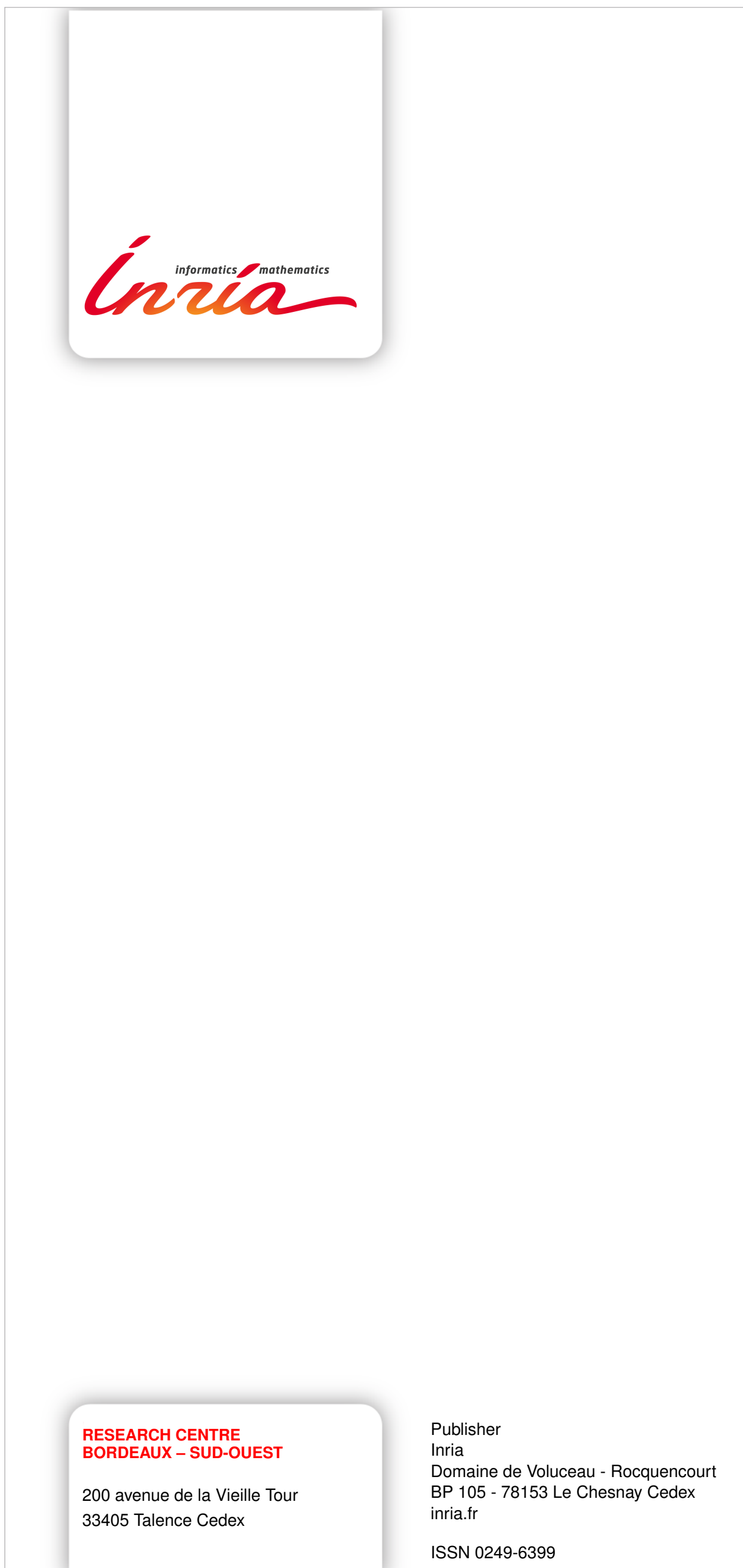

